\title{
Convergent abnormalities of $\beta$-amyloid deposition, glucose metabolism, and fMRI activity in the dorsal precuneus in subjective cognitive decline
}

Xuan-Yu Li ${ }^{1,6 a}$, M.D., Li-Xia Yuan ${ }^{2,7,8 a}$, Ph.D., Fei-Fan Zhou ${ }^{11}$, Ph.D., Chang-Chang

Ding $^{3}$, M.S., Teng-Fei Guo ${ }^{4}$, Ph.D., Wen-Ying Du ${ }^{1}$, M.D., Jie-Hui Jiang ${ }^{3}$, Ph.D.,

Frank Jessen ${ }^{5}$, M.D., Yu-Feng Zang ${ }^{2,7,8 a}$, M.D., Ph.D., Ying Han ${ }^{1,9,10,11^{*}}$, M.D., Ph.D.

1 Department of Neurology, Xuanwu Hospital of Capital Medical University, Beijing,

China, 100053.

2 Center for Cognition and Brain Disorders, The Affiliated Hospital of Hangzhou

Normal University, Hangzhou, China, 310015.

3 Department of Shanghai Institute for Advanced Communication and Data Science,

Shanghai University, Shanghai, China, 200444.

4 Institute of Biomedical Engineering, Shenzhen Bay Laboratory, Shenzhen, China, 518055.

5 Department of Psychiatry, Medical Faculty, University of Cologne, Cologne,

Germany, 50924.

6 Department of Neurology, Beijing Friendship Hospital, Capital Medical University, Beijing, China, 100050.

7 Zhejiang Key Laboratory for Research in Assessment of Cognitive Impairments, Hangzhou, China, 311121. 
medRxiv preprint doi: https://doi.org/10.1101/2021.04.14.21255317; this version posted April 20, 2021. The copyright holder for this preprint (which was not certified by peer review) is the author/funder, who has granted medRxiv a license to display the preprint in perpetuity. All rights reserved. No reuse allowed without permission.

8 Institutes of Psychological Sciences, Hangzhou Normal University, Hangzhou, China, 311121.

9 Center of Alzheimer's Disease, Beijing Institute for Brain Disorders, Beijing, China, 100053.

10 National Clinical Research Center for Geriatric Disorders, Beijing, China, 100053.

11 School of Biomedical Engineering, Hainan University, Haikou, China, 570228.

a: The authors contribute equally to this work.

\section{*Corresponding Author:}

Ying Han, Department of Neurology, Xuanwu Hospital of Capital Medical University,

Beijing, China, 100053. Email: hanying@xwh.ccmu.edu.cn. 


\section{Abstract}

\section{Background:}

There has been no report on convergent local abnormalities of multiple functional brain imaging modalities including $\beta$-amyloid $(A \beta)$ deposition, glucose metabolism, and resting-state functional magnetic resonance imaging (RS-fMRI) activities for participants with subjective cognitive decline (SCD).

\section{Methods:}

Fifty participants with SCD and 15 normal controls (NC) were scanned with both

$\left[{ }^{18} \mathrm{~F}\right]$-florbetapir positron emission tomography (PET) and $\left[{ }^{18} \mathrm{~F}\right]$-fluorodeoxyglucose PET, each PET sacn accompanied with simultaneous RS-fMRI. Voxel-wise metrics were analyzed, including $A \beta$ deposition, glucose metabolism, and three local metrics for RS-fMRI, i.e., amplitude of low frequency fluctuation (ALFF), regional homogeneity (ReHo), and degree centrality (DC).

\section{Results:}

The SCD group showed increased $A \beta$ deposition and increased glucose metabolism $(P<0.05$, corrected $)$, as well as decreased ALFF, ReHo, and DC $(P<0.05$, uncorrected) in the same area of the left dorsal precuneus $(\mathrm{dPCu})$. The $\mathrm{dPCu}$ showed negative resting state functional connectivity (RSFC) with the default mode network (DMN). Regarding global $A \beta$ deposition positivity, the $A \beta$ deposition in the $d P C u$ showed a gradient change, i.e., $\mathrm{SCD}^{+}>\mathrm{SCD}^{-}>\mathrm{NC}^{-}$. Further, both $\mathrm{SCD}^{+}$and $\mathrm{SCD}^{-}$ showed increased glucose metabolism and decreased RS-fMRI metrics in the $\mathrm{dPCu}$. 


\section{Conclusions:}

The convergent abnormal activities in the dPCu of SCD indicate that the $\mathrm{dPCu}$ is an early vulnerable region. The anti-RSFC of the $\mathrm{dPCu}$ with DMN supports that the earliest symptoms might be more related to other cognitive functions (e.g., unfocused attention) than episodic memory. (Funded by the National Key Research and Development Program of China and others; ClinicalTrials.gov number, NCT03370744.)

\section{Introduction}

Subjective cognitive decline (SCD) refers to self-perceived cognitive decline relative to a previous status in the absence of measurable objective cognitive impairment. ${ }^{1,2}$ SCD may be the first symptomatic manifestation of Alzheimer's disease $(\mathrm{AD})$ and indicate a higher risk for a future objective cognitive impairment. ${ }^{1}$ Functional neuroimaging techniques, including $\beta$-amyloid $(A \beta)$ positron emission tomography (PET), tau protein PET, $\left[{ }^{18} \mathrm{~F}\right]$-fluorodeoxyglucose (FDG)-PET, and resting-state functional magnetic resonance imaging (RS-fMRI), have been used in SCD studies. However, as summarized in the Supplementary Appendix (SA) Table $\mathrm{S} 1$, there has been no consensus on where the abnormal activity is in SCD.

The current study used a PET/MR hybrid scanner and analyzed the local activities including $\mathrm{A} \beta$ deposition, glucose metabolism, and three RS-fMRI local metrics, i.e., amplitude of low frequency fluctuation (ALFF), regional homogeneity (ReHo), and

degree centrality (DC) in SCD and normal control (NC) ${ }^{3-5}$ Previous coordinate-based 
meta-analytic studies have suggested that the posterior medial parietal area in the default mode network (DMN) regions including the posterior cingulate cortex (PCC) and its adjacent precuneus ( $\mathrm{PCu}$ ) (usually named $\mathrm{PCC} / \mathrm{PCu}$ in literatures) consistently showed increased $A \beta$ deposition, decreased glucose metabolism, decreased ALFF, and decreased ReHo in the mild cognitive impairment (MCI) or AD. ${ }^{6-9}$ We therefore hypothesized that the PCC/PCu in SCD would show convergent abnormal activities.

\section{Methods}

\section{Participants}

Participants were recruited through public advertisements and referrals from general physicians, memory clinics, and informants. All participants signed a written informed consent before the experiment. The recruitment and assessment of participants have been described in the protocol of the Sino Longitudinal Study on Cognitive Decline (SILCODE) previously. ${ }^{10}$ The study was registered in ClinicalTrials.gov (Identifier: NCT03370744) and approved by the ethics committee of Xuanwu Hospital of Capital Medical University (Identifier No. [2017]046). The participants were recruited from February 27th to November 15th in 2018, and finally 65 right-handed and Mandarin speaking participants (50 for SCD and 15 for NC) were included. Fig. S1 illustrates the eligibility criteria for the study population.

SCD was defined according to the research criteria for pre-MCI SCD, including 1) self-experienced persistent decline in cognitive capacity compared with a previously normal status and unrelated to an acute event; 2) normal age-, sex-, and 
education-adjusted performance on standardized cognitive tests and not meet the criteria for MCI or dementia. ${ }^{1,10}$ Individuals with normal performance on the standardized neuropsychological tests and without cognitive problems via the SCD-interview ${ }^{11}$ were included as the NC group.

The exclusion criteria were as follows: 1) a history of stroke; 2) current major psychiatric diagnoses, such as severe depression and anxiety (Hamilton depression scale (HAMD) score > 24 or Hamilton anxiety (HAMA) scale > 29); 3) other diseases that could cause cognitive decline; 4) history of psychosis or congenital mental developmental delay; 5) contraindications for MRI or unable to complete the study protocol.

\section{Neuropsychological assessments}

The neuropsychological tests included three cognitive domains: auditory verbal learning test-Huashan version (AVLT-H), animal fluency test (AFT), 30-item Boston naming test (BNT), and shape trail test (STT) parts A and B. ${ }^{12-15}$ In addition, all subjects implemented tests for global cognition, daily life ability, and neuropsychiatric assessments, including Montreal cognitive assessment-basic (MoCA-B), ${ }^{16}$ functional activities questionnaire (FAQ), HAMA, and HAMD.

\section{Image data acquisition}

For each participant, PET and MRI data were simultaneously acquired with an integrated 3.0 Tesla TOF PET/MR scanner (SIGNA PET/MR, GE Healthcare, 
Milwaukee, WI, USA) at Xuanwu Hospital of Capital Medical University. Participants were instructed to keep their eyes closed, relax but not fall asleep, and move as little as possible during scanning. Foam pads were used to minimize head movements and earplugs were used to reduce machine noise.

\section{PET data acquisition}

Participants were scanned with $\left[{ }^{18} \mathrm{~F}\right]$ florbetapir (AV45) and FDG-PET in a 3D acquisition mode about four weeks apart (24.4 \pm 23.8 days). For FDG-PET, participants were instructed to fast for at least $6 \square$ hours. The imaging parameters were listed in the Method-SA.

\section{MRI data acquisition}

We obtained two fMRI datasets for each participant, namely AV45-fMRI and FDG-fMRI. The detailed scanning parameters for the 3D-T1 and RS-fMRI images were in the Method-SA.

\section{Data analysis}

AV45-PET and FDG-PET images were analyzed by Statistical Parametric Mapping version 12 (SPM 12; https://www.fil.ion.ucl.ac.uk/spm/software/spm12) and in-house software with MATLAB (https://www.mathworks.com). The preprocessing and calculation of the standardized uptake value ratio (SUVR) of the AV45-PET (voxel-wise and global SUVR) and FDG-PET (voxel-wise SUVR) were listed in the Method-SA. 
RS-fMRI datasets were preprocessed using the Data Processing Assistant for Brain Imaging (DPABI_V4.1) and Resting-State fMRI Data Analysis Toolkit (RESTplus_1.22). ${ }^{17,18}$ The details were listed in the Method-SA.

Then, three RS-fMRI metrics for local activities were calculated, including ALFF reflecting the amplitude of the fluctuation of each time series of the fMRI signal, ReHo reflecting the local synchronization of the time series of neighboring voxels, and DC reflecting the functional connectivity density of a voxel with all other voxels. $^{3-5}$ It should be noted that, DC is actually an FC index, but often taken as a local metric in graph theory analysis.

Seed-based FC analysis was a post-hoc analysis. As shown in the Results section, 6 voxels in the left dorsal precuneus ( $\mathrm{dPCu}$ ), which showed abnormal activities in 7 of the total 8 neuroimaging metrics in SCD subjects, were taken as a region of interest (named dPCu-ROI hereafter). The details for FC analysis were listed in the Method-SA.

\section{Statistical analyses}

SPSS (version 24.0, IBM) was used for comparisons of demographic and neuropsychological data. DPABI_V4.1 was used for statistical analysis of the local activities and FC between SCD and NC. In addition, the A $\beta$ positivity effect was also tested. ${ }^{19}$ The details were listed in the Method-SA. 


\section{Results}

\section{Behavioral measures}

No significant difference was found in the age, sex, or education level between the SCD group and NC group (all $P>0.05$ ) (Table 1). The HAMA and HAMD were significantly higher in the SCD group than in the NC group.

\section{Alteration of local activity}

The SCD group showed: 1) increased $\mathrm{A} \beta$ deposition in the left $\mathrm{dPCu}$, left cuneus, left superior parietal cortex, and right medial superior frontal cortex (Figure 1. $P<$ 0.05, FDR corrected); 2) increased glucose metabolism in the left dPCu, left middle occipital cortex, and bilateral paracentral lobule (Figure 1. $P<0.05$, FDR corrected). The left $\mathrm{dPCu}$ was the only region showing overlapped abnormality for $\mathrm{A} \beta$ deposition and glucose metabolism.

The difference of RS-fMRI metrics (ALFF, ReHo, and DC) did not survive FDR correction. With an uncorrected $P<0.05$, the SCD group showed decreased ALFF, ReHo, and DC in the left dPCu (Figure 1) for both the AV45-fMRI and FDG-fMRI.

\section{Seed-based functional connectivity}

One sample $t$-tests showed that the connectivity patterns of the left $\mathrm{dPCu}$ were very similar for AV45-fMRI and FDG-fMRI datasets $(P<0.05$, FDR corrected, Figure 2$)$. Significantly negative functional connectivity with the left $\mathrm{dPCu}$ was mainly in the 
DMN regions, including the bilateral medial prefrontal cortex, posterior cingulate cortex, and its adjacent ventral PCu, etc. Significantly positive functional connectivity was located in the bilateral inferior frontal cortex, middle frontal cortex, lingual gyrus, etc. No significant difference in RSFC was found between the SCD group and NC group $(P<0.05$, FDR corrected).

\section{Correlation between imaging metrics and behavioral measures in the SCD group}

We observed significant correlation between any pair of two fMRI metrics (ALFF,

ReHo, and DC; $P<0.001)$ within the dPCu-ROI. No significant correlation was found between other pairs of imaging metrics (Table S2).

As for the correlation between behavioral scales and imaging metrics, although a few correlation showed a $P$ value between $0.05-0.01$, none survided multiple comarision correction (Table S3).

\section{A $\beta$ deposition positivity and neuroimaging metrics in the dPCu-ROI}

The demographic and neuropsychological data of $\mathrm{NC}^{-}, \mathrm{SCD}^{-}$and $\mathrm{SCD}^{+}$groups can be found in Table S4. The HAMD score was significantly higher in the $\mathrm{SCD}^{+}$group and the $\mathrm{SCD}^{-}$group than in the $\mathrm{NC}^{-}$group.

$\mathrm{SCD}^{+}$group showed significantly higher global AV45-SUVR than both $\mathrm{NC}^{-}$and $\mathrm{SCD}^{-}$groups, but no significant difference was found between $\mathrm{NC}^{-}$and $\mathrm{SCD}^{-}(P=$

0.43) (Figure 3A). Both $\mathrm{SCD}^{-}$and $\mathrm{SCD}^{+}$groups had significantly higher AV45-SUVR in left dPCu-ROI than NC-group, while no significant difference was 
found between $\mathrm{SCD}^{-}$and $\operatorname{SCD}^{+}(P=0.08)$ (Figure 3B).With regard to the FDG-SUVR and all RS-fMRI metrics, both $\mathrm{SCD}^{+}$and $\mathrm{SCD}^{-}$showed hypermetabolism and decreased fMRI activity in the dPCu-ROI, except for the comparison of FDG-ReHo between $\mathrm{SCD}^{+}$group and $\mathrm{NC}^{-}$group $(P=0.11)$ (Figure 3).

\section{Independent verification of data analyses}

In order to controlling for the potential influence from data anslysis upon our results, we replicated all the analyses by two independent research teams based on the same software and pipeline. The results were substaitially the same (Fig. S2-4 and Table S5-6).

\section{Discussion}

\section{Convergent abnormal local activity in AD/MCI/SCD}

In the PCC and its adjacent $\mathrm{PCu}$ (sometimes named $\mathrm{PCC} / \mathrm{PCu}$ ) of $\mathrm{MCI}$ and $\mathrm{AD}$, meta-analyses have reported increased $A \beta$ deposition and decreased glucose metabolism, decreased RS-fMRI ALFF, and ReHo. ${ }^{6,8,9}$ However, the current study found convergent abnormal activities in 7/8 functional metrics in the $\mathrm{dPCu}$. Furthermore, the seed-based RS-fMRI FC showed that the dPCu was negatively connected with the DMN (including PCC and its adjacent ventral PCu). Previous RS-fMRI studies have segmented the precuneus into 6 or 8 sub-regions, ${ }^{20,21}$ suggesting precuneus may be functionally heterogeneous. We searched in the Neurosynth (http://neurosynth.org) (coordinates: -6, -68, 62; distance: $4 \mathrm{~mm}$ ) and 
found that the $\mathrm{dPCu}$ was reported to be involved in more cognitive than memory tasks, e.g., unfocused attention, ${ }^{22}$ in healthy people. Although many task fMRI studies have been carried out on SCD (Table S7), none has reported abnormal activation in the $\mathrm{dPCu}$. Future studies should investigate the specific cognitive processing of the dPCu, and hence develop more specific cognitive tests for SCD.

\section{Increased A $\beta$ deposition vs. increased glucose metabolism in SCD}

Meta-analysis has reported that the increased $A \beta$ deposition was associated with decreased glucose metabolism in the same area of PCC in MCI/AD, ${ }^{6}$ indicating a decreased neuronal activity. However, we found an association of increased $A \beta$ deposition with increased glucose metabolism in the $\mathrm{dPCu}$, indicating an increased activity. No previous PET imaging studies of SCD, MCI and AD reported one positive relationship between cerebral $A \beta$ deposition and glucose metabolism as we found in this study. A few human PET and task fMRI studies ${ }^{23-25}$ indirectly support our findingsStudies of animal model of early $\mathrm{AD}$ have frequently reported A $\beta$-dependent increased neuronal activity. ${ }^{26,27}$ Our results support the view that $A \beta$-dependent hyperactivity may occur long before the presence of $\mathrm{AD}$ symptoms. ${ }^{26}$

\section{Decreased value of RS-fMRI local metrics vs. increased glucose metabolism in}

\section{SCD}

As aforementioned, for MCI and $\mathrm{AD}$, both the decreased glucose metabolism and decreased value of RS-fMRI local metrics (ALFF and ReHo) in the PCC were interpreted as decreased neuronal activity. However, we found seemingly discrepant 
results, i.e., increased glucose metabolism and decreased value of the local RS-fMRI metrics in the dPCu. The following notations might be helpful.

1) Mathematically, the glucose metabolism of FDG-PET reflects the integrated or mean value over a period of time, while the RS-fMRI ALFF reflects the amplitude of fluctuation or standard deviation within a specific frequency band. The simulation result showed that the mean value has no linear correlation with the standard deviation (Fig. S5). It has commonly been suggested that the fMRI signal is more coupled with the local field potential (LFP) than the action potential. ${ }^{28}$ Mathematically, both the RS-fMRI signal and the LFP are analyzed in a frequency-dependent way, however, their frequency-dependent correlation of the amplitude, a popular metric for both RS-fMRI and LFP, is far from clear.

2) A few PET-fMRI studies have shown that, although both the glucose metabolism $^{29}$ and RS-fMRI local metrics show similar higher activity in the default mode network (DMN) regions (e.g., PCC) than other brain regions, voxel-wise across-subject correlation did not show robust significant linear correlation (Table $\mathrm{S} 8)^{30-32}$

3) Brain disorders may show opposite association of glucose metabolism with RS-fMRI local metrics, e.g., increased glucose metabolism ${ }^{33}$ but decreased $\mathrm{ALFF}^{34}$ in the putamen of patients with Parkinson's disease (Table S9). The current study also showed opposite association in the $\mathrm{dPCu}$, however, as aforementioned, $\mathrm{AD} / \mathrm{MCI}$ showed positive association, i.e., decreased glucose metabolism and decreased value of RS-fMRI metrics. ${ }^{6,8,9}$ 
Considering the non-invasiveness, low-cost, and easy-accessing advantages of RS-fMRI technique, it may be a promising technique for precise localizing the AD-related abnormal activity, and hence, could be used to guide precise brain stimulation, e.g., transcranial magnetic stimulation.

\section{SCD showed abnormal local activities regardless of A $\beta$ positivity}

Binary classification of $\mathrm{A} \beta$ deposition into positive and negative is a popular way for clinical diagnosis for either healthy aging or participants with SCD. ${ }^{35}$ The current study thus classified all participants into $\mathrm{SCD}^{+}, \mathrm{SCD}^{-}, \mathrm{NC}^{+}$, and $\mathrm{NC}^{-}$with the criteria of the cortical summary AV45-SUVR value $\geq 1.11 .{ }^{19} \mathrm{NC}^{+}$group was not included into the further analysis due to the limited sample size $(n=4)$. Both the $\mathrm{SCD}^{-}$and $\mathrm{SCD}^{+}$groups showed increased $\mathrm{A} \beta$ deposition and glucose metabolism and decreased value of RS-fMRI metrics in the $\mathrm{dPCu}$ as compared with $\mathrm{NC}^{-}$group. In other words, like the $\mathrm{SCD}^{+}$group, the $\mathrm{SCD}^{-}$group also showed evidences of elevated $\mathrm{A} \beta$ pathology and hypermetabolism, as well as abnormal fMRI activities. Although the $\mathrm{A} \beta+/$ - categorization is often used to define preclinical $\mathrm{AD}$, it does not imply that gobally $A \beta$ negative subjects have no pathology in the brain. ${ }^{35,36}$ Consistent with our findings, previous literature also found that local regions (including precuneus and posterior cingulate etc.) may be more sensitive to detect early $\mathrm{A} \beta$ deposition than global cortical summary regions. ${ }^{37-39}$ Furthermore, we also compared the mean values of the eight metrics in the PCC (Fig. S6), the $\mathrm{SCD}^{+}$group showed significantly higher AV45-SUVR than the $\mathrm{SCD}^{-}$and $\mathrm{NC}^{-}$group. However, we did not found significant difference in the FDG-SUVR and fMRI metrics of PCC between the NC-group and 
the two SCD groups. Together, it is likely that the increased A $\beta$ deposition may occur concurrently with hypermetabolism and abnormal fMRI activities in dPCu (early $\mathrm{A} \beta$-affected region) in the early stage of AD.

\section{Limitations}

This study has some limitations. First, this is a cross-sectional data, thus further longitudinal studies would be helpful to investigate the changes of $\mathrm{A} \beta$ deposition, neuronal dysfunction, and cognitive function as the disease progresses. Second, we did not have tau PET imaging, thus we can not evaluate tau pathology in this study. Third, although multimodal metrics and two fMRI datasets were used to increase the reliability of our results, data from multiple research centers are needed for validation of the findings in this study. Finally, this study did not include cognitive tests related to unfocused attention which might be more specific to elaborate the functional roles of the $\mathrm{dPCu}$.

\section{Conclusions}

In summary, we found increased $A \beta$ deposition, increased glucose metabolism, and decreased value of RS-fMRI metrics in the same area of the left $\mathrm{dPCu}$ in SCD. The negative functional connectivity between the left $\mathrm{dPCu}$ and DMN suggests that the $\mathrm{dPCu}$ is not a region in the DMN. The left $\mathrm{dPCu}$ may be among the earliest impaired regions, and the DMN is probably impaired in a later stage, e.g., MCI/AD. Precisely targeting on the left $\mathrm{dPCu}$, future studies should develop delicate cognitive tasks to probe the cognitive impairments by task fMRI and/or virtual lesion of 
transcranial magnetic stimulation (TMS). Further, repetitive TMS could be used as an early intervention on the $\mathrm{dPCu}$.

Funding This work was supported by the National Natural Science Foundation of China (61633018, 82020108013 and 81661148045).

Data Availability Statement The dataset generated and analyzed in the current study is available fromthe corresponding author on reasonable request.

Conflict of Interest: The authors declare that the research was conducted in the absence of any commercial or financial relationships that could be construed as a potential conflict of interest.

\section{References}

1. Jessen F, Amariglio RE, van Boxtel M, et al. A conceptual framework for research on subjective cognitive decline in preclinical Alzheimer's disease. Alzheimers Dement 2014;10:844-852.

2. Molinuevo JL, Rabin LA, Amariglio R, et al. Implementation of subjective cognitive decline criteria in research studies. Alzheimers Dement 2017;13:296-311.

3. Zang YF, He Y, Zhu CZ, et al. Altered baseline brain activity in children with ADHD revealed by resting-state functional MRI. Brain Dev 2007;29:83-91. 
4. Zang Y, Jiang T, Lu Y, He Y, Tian L. Regional homogeneity approach to fMRI data analysis. Neuroimage 2004;22:394-400.

5. Zuo XN, Ehmke R, Mennes M, et al. Network centrality in the human functional connectome. Cereb Cortex 2012;22:1862-1875.

6. He W, Liu D, Radua J, Li G, Han B, Sun Z. Meta-analytic comparison between PIB-PET and FDG-PET results in Alzheimer's disease and MCI. Cell Biochem Biophys 2015;71:17-26.

7. Reiman EM, Caselli RJ, Yun LS, et al. Preclinical evidence of Alzheimer's disease in persons homozygous for the epsilon 4 allele for apolipoprotein E. $\mathrm{N}$ Engl J Med 1996;334:752-758.

8. Pan P, Zhu L, Yu T, et al. Aberrant spontaneous low-frequency brain activity in amnestic mild cognitive impairment: A meta-analysis of resting-state fMRI studies. Ageing Res Rev 2017;35:12-21.

9. Zheng D, Xia W, Yi ZQ, et al. Alterations of brain local functional connectivity in amnestic mild cognitive impairment. Transl Neurodegener 2018;7:26.

10. Li X, Wang X, Su L, Hu X, Han Y. Sino longitudinal study on cognitive decline (SILCODE): protocol for a Chinese longitudinal observational study to develop risk prediction models of conversion to mild cognitive impairment in individuals with subjective cognitive decline. BMJ Open 2019;9:e028188.

11. Jessen F, Spottke A, Boecker H, et al. Design and first baseline data of the DZNE multicenter observational study on predementia Alzheimer's disease (DELCODE). Alzheimers Res Ther 2018;10:15. 
12. Zhao Q, Lv Y, Zhou Y, Hong Z, Guo Q. Short-term delayed recall of auditory verbal learning test is equivalent to long-term delayed recall for identifying amnestic mild cognitive impairment. PLoS One 2012;7:e51157.

13. Guo Q, Jin L, Hong Z, Lv C. A specific phenomenon of animal fluency test in Chinese elderly. Chinese Mental Health Journal 2007;21:622-625.

14. Guo Q, Hong Z, Shi W, Sun Y, Lv C. Boston naming test in Chinese elderly, patient with mild cognitive impairment and Alzheimer's dementia. Chinese Mental Health Journal 2006;20:81-84.

15. Zhao Q, Guo Q, Li F, Zhou Y, Wang B, Hong Z. The Shape Trail Test: application of a new variant of the Trail making test. PLoS One 2013;8:e57333.

16. Chen KL, Xu Y, Chu AQ, et al. Validation of the Chinese Version of Montreal Cognitive Assessment Basic for Screening Mild Cognitive Impairment. J Am Geriatr Soc 2016;64:e285-e290.

17. Yan CG, Wang XD, Zuo XN, Zang YF. DPABI: Data Processing \& Analysis for (Resting-State) Brain Imaging. Neuroinformatics 2016;14:339-351.

18. Jia XZ, Wang J, Sun HY, et al. RESTplus:an improved toolkit for resting-state functional magnetic resonance imaging data processing. Sci bull 2019;64:953-954.

19. Landau SM, Breault C, Joshi AD, et al. Amyloid- $\beta$ imaging with Pittsburgh compound B and florbetapir: comparing radiotracers and quantification methods. J Nucl Med 2013;54:70-77. 
20. Luo Z, Zeng LL, Qin J, Hou C, Shen H, Hu D. Functional Parcellation of Human Brain Precuneus Using Density-Based Clustering. Cereb Cortex 2020;30:269-282.

21. Zhang S, Li CS. Functional connectivity mapping of the human precuneus by resting state fMRI. Neuroimage 2012;59:3548-62.

22. Dickenson J, Berkman ET, Arch J, Lieberman MD. Neural correlates of focused attention during a brief mindfulness induction. Soc Cogn Affect Neurosci $2013 ; 8: 40-47$

23. Cohen AD, Price JC, Weissfeld LA, et al. Basal cerebral metabolism may modulate the cognitive effects of Abeta in mild cognitive impairment: an example of brain reserve. J Neurosci 2009;29:14770-14778.

24. Oh H, Madison C, Baker S, Rabinovici G, Jagust W. Dynamic relationships between age, amyloid- $\beta$ deposition, and glucose metabolism link to the regional vulnerability to Alzheimer's disease. Brain 2016;139:2275-2289.

25. Sperling RA, Dickerson BC, Pihlajamaki $M$, et al. Functional alterations in memory networks in early Alzheimer's disease. Neuromolecular Med $2010 ; 12: 27-43$

26. Zott B, Busche MA, Sperling RA, Konnerth A. What happens with the circuit in Alzheimer's disease in mice and humans? Annu Rev Neurosci 2018;41:277-297.

27. Zott B, Simon MM, Hong W. A vicious cycle of $\beta$ amyloid-dependent neuronal hyperactivation. Science 2019;365:559-565. 
28. Logothetis NK, Pauls J, Augath M, Trinath T, Oeltermann A. Neurophysiological investigation of the basis of the fMRI signal. Nature 2001;412:150-157.

29. Raichle ME, MacLeod AM, Snyder AZ, Powers WJ, Gusnard DA, Shulman GL. A default mode of brain function. Proc Natl Acad Sci U S A 2001;98(2):676-682.

30. Aiello M, Salvatore E, Cachia A, et al. Relationship between simultaneously acquired resting-state regional cerebral glucose metabolism and functional MRI: A PET/MR hybrid scanner study. Neuroimage 2015;113:111-121.

31. Bernier M, Croteau E, Castellano CA, Cunnane SC, Whittingstall K. Spatial distribution of resting-state BOLD regional homogeneity as a predictor of brain glucose uptake: A study in healthy aging. Neuroimage 2017;150:14-22.

32. Jiao F, Gao Z, Shi K, et al. Frequency-dependent relationship between resting-state fMRI and glucose metabolism in the elderly. Front Neurol 2019;10:566.

33. Tomše $\mathrm{P}$, Jensterle L, Grmek M, et al. Abnormal metabolic brain network associated with Parkinson's disease: replication on a new European sample. Neuroradiology 2017;59:507-515.

34. Wang J, Zhang JR, Zang YF, Wu T. Consistent decreased activity in the putamen in Parkinson's disease: a meta-analysis and an independent validation of resting-state fMRI. Gigascience 2018;7:giy071.

35. Jack CR Jr, Bennett DA, Blennow K, et al. A/T/N: An unbiased descriptive classification scheme for Alzheimer disease biomarkers. Neurology 2016;87:539-47. 
36. Jack CR Jr, Bennett DA, Blennow K, et al. NIA-AA Research Framework: Toward a biological definition of Alzheimer's disease. Alzheimers Dement 2018;14:535-562.

37. Palmqvist S, Schöll M, Strandberg O, et al. Earliest accumulation of $\beta$-amyloid occurs within the default-mode network and concurrently affects brain connectivity. Nat Commun 2017;8:1214.

38. Farrell ME, Chen X, Rundle MM, Chan MY, Wig GS, Park DC. Regional amyloid accumulation and cognitive decline in initially amyloid-negative adults. Neurology 2018;91:e1809-e1821.

39. Guo T, Landau SM, Jagust WJ; Alzheimer's Disease Neuroimaging Initiative. Detecting earlier stages of amyloid deposition using PET in cognitively normal elderly adults. Neurology 2020;94:e1512-e1524. 


\section{Figure legends}

Figure 1. Group differences between subjective cognitive decline (SCD) and normal control (NC) groups with controlling for age, sex, and years of education, on $\mathrm{A} \beta$ deposition and glucose metabolism (top row), RS-fMRI metrics (middle row), and the overlapping area of the PET and fMRI metrics (bottom row). Overlapping area at the left $\mathrm{dPCu}$ means that 7 of 8 metrics showed group difference, i.e., AV45-SUVR, FDG-SUVR, AV45-ALFF, FDG-ALFF, AV45-ReHo, FDG-ReHo, AV45-DC, and FDG-DC. Red and yellow colors indicate increased metrics in SCD vs. NC, and blue color indicates the opposite. SUVR: standardized uptake value ratio. FDR: false discovery rate. ALFF: amplitude of low frequency fluctuation. ReHo: regional homogeneity. DC: degree centrality. L and R: left and right in the brain. $T$ : the $T$ value of two-sample $t$-test on the PET and fMRI metrics maps.

Figure 2. Patterns of seed-based resting-state functional connectivity (RSFC) of the left dorsal precuneus ( $\mathrm{dPCu}$ ) of all the subjects in the AV45-fMRI dataset (A) and FDG-fMRI dataset $(\mathrm{B})(\mathrm{P}<0.05$, FDR corrected). The yellow-red color indicates positive connections and the blue color indicates negative connections with the left dPCu. MPFC: medial prefrontal cortex. PCC: posterior cingulate cortex. vPCu: ventral PCu. IPS: intraparietal sulcus. L and R: left and right in the brain. $T$ : the $T$ value of one-sample $t$-test on the RSFC maps. 
medRxiv preprint doi: https://doi.org/10.1101/2021.04.14.21255317; this version posted April 20, 2021. The copyright holder for this preprint (which was not certified by peer review) is the author/funder, who has granted medRxiv a license to display the preprint in perpetuity. All rights reserved. No reuse allowed without permission.

Figure 3. Comparisons of global AV45-SUVR (A) and the 8 metrics in the left dPCu-ROI among three groups, i.e. AV45-SUVR (B), FDG-SUVR (C), AV45-ALFF (D), AV45-ReHo (E), AV45-DC (F), FDG-ALFF (G), FDG-ReHo (H), and FDG-DC (I). $\mathrm{NC}^{-}: \mathrm{A} \beta$ negative $\mathrm{NC} . \mathrm{SCD}^{-}: \mathrm{A} \beta$ negative $\mathrm{SCD} . \mathrm{SCD}^{+}: \mathrm{A} \beta$ positive $\mathrm{SCD} . *: P<$ $0.05, * *: P<0.01, * * *: P<0.001$. All box and whisker plots: box range, $25-75 \%$; whisker range, $5-95 \%$. 
Table 1. Demographic information and neuropsychological tests.

\begin{tabular}{|c|c|c|c|}
\hline & $\operatorname{SCD}(\mathrm{N}=50)$ & $\mathrm{NC}(\mathrm{N}=15)$ & Statistics \\
\hline Age (years) & $66.4 \pm 5.7$ & $66.3 \pm 4.4$ & $t_{(63)}=0.04, P=0.97$ \\
\hline Gender (M/F) & $19 / 31$ & $5 / 10$ & $\chi_{(1)}^{2}=0.11, P=0.74$ \\
\hline Education (years) & $13.8 \pm 3.0$ & $12.8 \pm 2.5$ & $t_{(63)}=1.16, P=0.25$ \\
\hline HAMD & $3.8 \pm 3.7$ & $1.1 \pm 1.3$ & $t_{(63)}=2.81, P=0.007$ \\
\hline HAMA & $4.5 \pm 3.9$ & $2.1 \pm 2.4$ & $t_{(63)}=2.25, P=0.03$ \\
\hline FAQ & $0.2 \pm 0.5$ & $0.0 \pm 0.0$ & $t_{(61)}=1.63, P=0.11^{\#}$ \\
\hline AVLT-H delayed recall & $7.7 \pm 2.0$ & $7.3 \pm 1.9$ & $t_{(63)}=0.70, P=0.48$ \\
\hline AVLT-H recognition & $22.3 \pm 1.4$ & $22.5 \pm 1.6$ & $t_{(63)}=-0.30, P=0.77$ \\
\hline STT part A & $55.5 \pm 17.7$ & $53.2 \pm 14.5$ & $t_{(63)}=0.46, P=0.65$ \\
\hline STT part B & $128.7 \pm 37.0$ & $128.5 \pm 29.1$ & $t_{(63)}=0.03, P=0.98$ \\
\hline AFT & $20.0 \pm 4.7$ & $19.5 \pm 4.8$ & $t_{(63)}=0.40, P=0.69$ \\
\hline BNT & $26.0 \pm 2.4$ & $25.5 \pm 3.2$ & $t_{(63)}=0.74, P=0.46$ \\
\hline MoCA-B & $26.3 \pm 2.1$ & $26.1 \pm 1.9$ & $t_{(63)}=0.46, P=0.65$ \\
\hline
\end{tabular}

Data were presented as mean \pm SD. SCD: subjective cognitive decline. NC: normal control.

HAMD: Hamilton depression scale. HAMA: Hamilton anxiety scale. FAQ: functional activities questionnaire. AVLT-H: auditory verbal learning test-Huashan version. STT: shape trails test. AFT: animal fluency test. BNT: Boston naming test. MoCA-B: Montreal cognitive assessment-basic. " : Missing data for 1 SCD and 1 NC subjects. 


\section{AV45-PET}

\section{SUVR}

FDR $P<0.05$

\section{$L \frac{z=36 \quad z=45}{A V 45-f M R I}$}

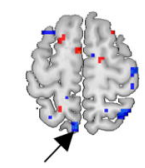

$$
z=41 \quad z=61 \quad z=66
$$

\section{ReHo}

$$
P<0.05
$$

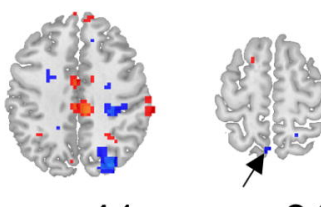

$$
z=41 \quad z=61 \quad z=66
$$

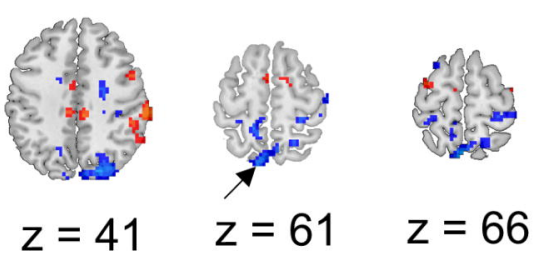

\section{FDG-PET}
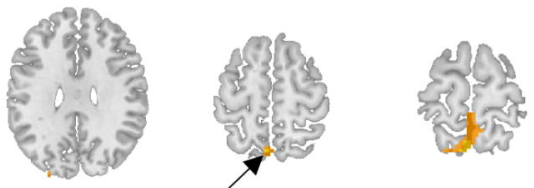

$z=30$
$\mathrm{R}$

FDG-fMRI
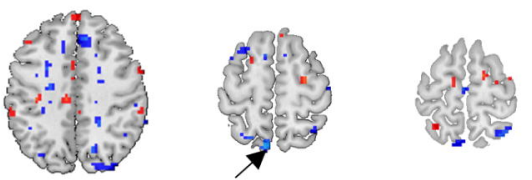

$$
z=41
$$

$z=61$

$z=66$
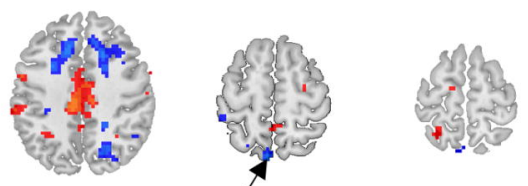

$$
z=41
$$

$z=61$

$z=66$

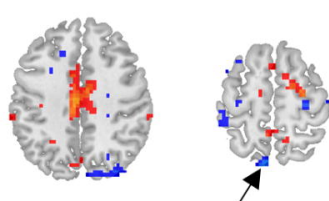

$P<0.05$

$$
z=41
$$

$z=41$

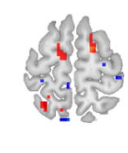

$z=66$

Overlapping area

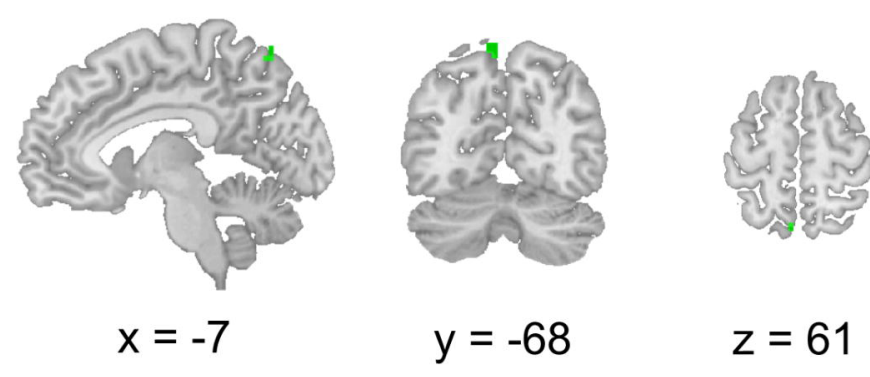




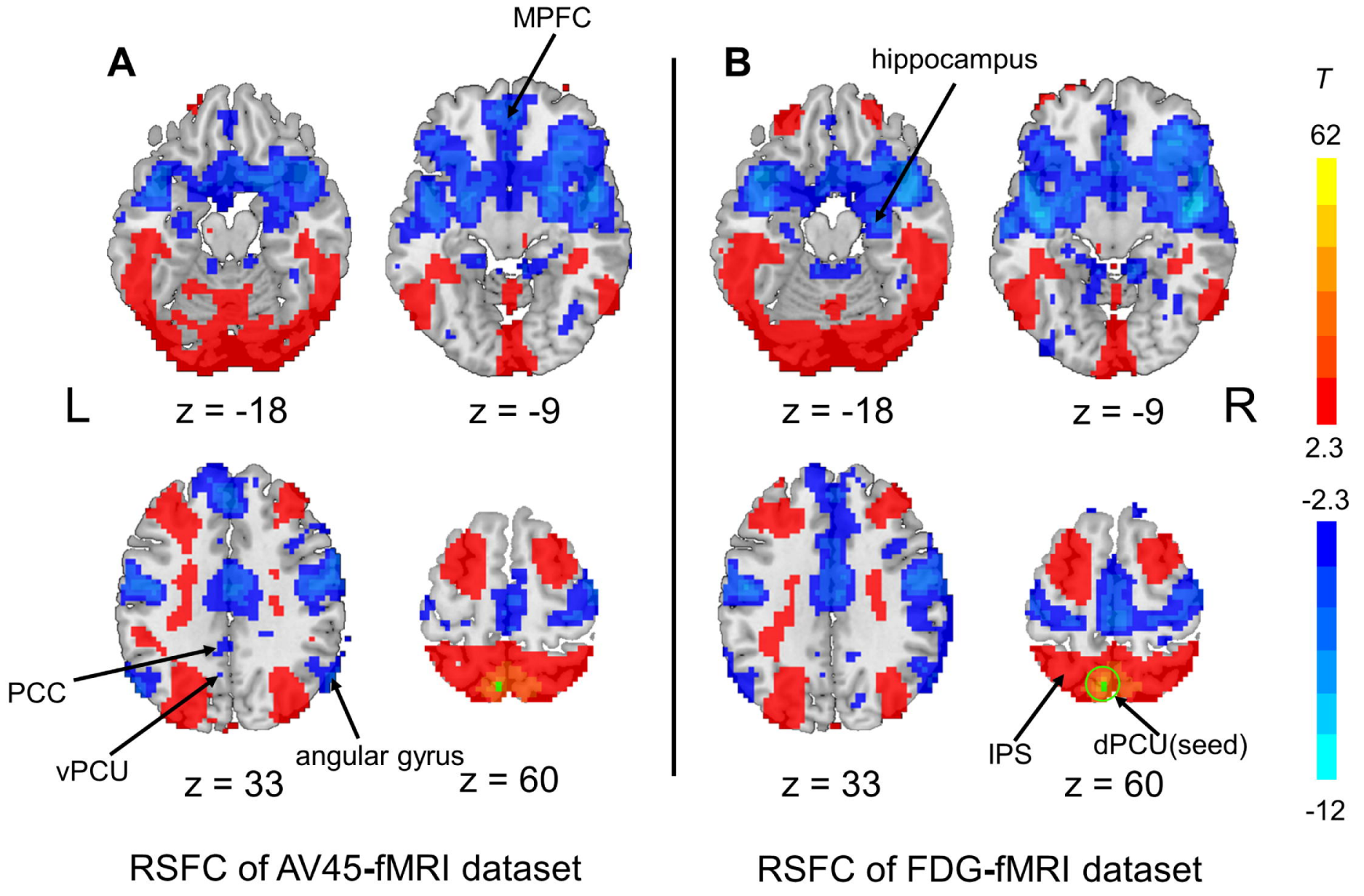



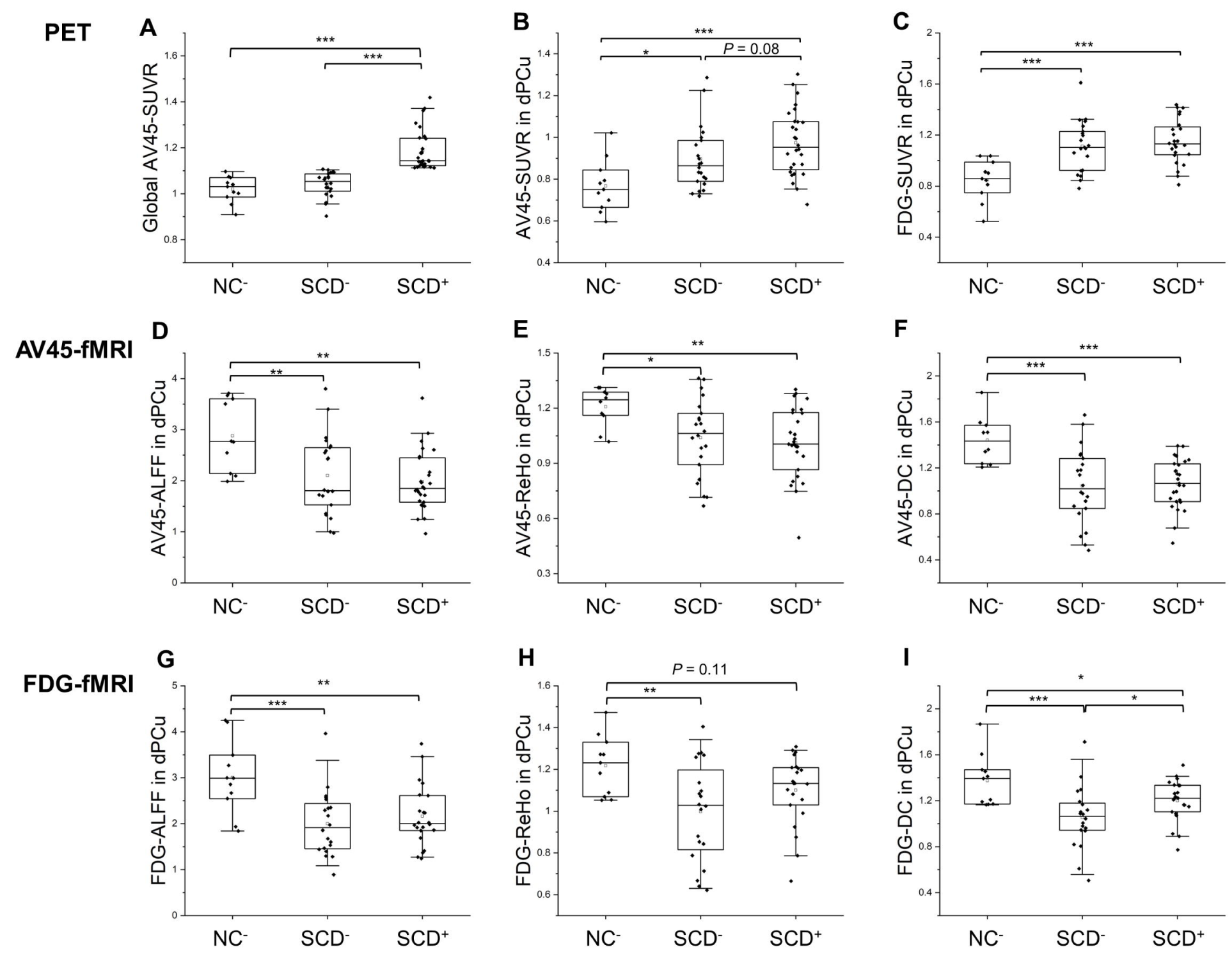